\title{
Density profile of a Bose-Einstein condensate inside a pancake-shaped trap: observational consequences of the dimensional cross-over in the scattering properties
}

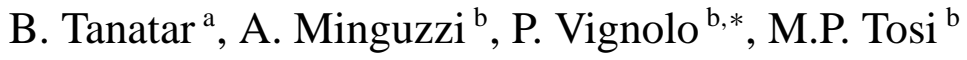 \\ a Department of Physics, Bilkent University, Bilkent, 06533 Ankara, Turkey \\ ${ }^{\mathrm{b}}$ INFM and Classe di Scienze, Scuola Normale Superiore, I-56126 Pisa, Italy
}

Received 27 June 2002; accepted 25 July 2002

Communicated by V.M. Agranovich

\begin{abstract}
It is theoretically well known that two-dimensionality of the scattering events in a Bose-Einstein condensate introduces a logarithmic dependence on density in the coupling constant entering a mean-field theory of the equilibrium density profile, which becomes dominant as the $s$-wave scattering length gets larger than the condensate thickness. We trace the regions of experimentally accessible system parameters for which the cross-over between different dimensionality behaviors in the scattering properties may become observable through in situ imaging of the condensed cloud with varying trap anisotropy and scattering length.

(c) 2002 Elsevier Science B.V. All rights reserved.
\end{abstract}

PACS: 03.75.Fi; 05.30.Jp; 32.80.Pj

Keywords: Bose gases; Low dimensional; Density profiles

\section{Introduction}

A dilute fluid of hard-disk bosons constrained to move inside a two-dimensional (2D) box is a wellknown model in statistical mechanics [1]. This system shows very different features from its threedimensional (3D) counterpart. First of all its collisional properties are very peculiar, since in two dimensions the T-matrix vanishes at low momentum and

\footnotetext{
* Corresponding author

E-mail address: vignolo@sns.it (P. Vignolo).
}

energy $[1,2]$ and the resulting boson-boson coupling constant depends on the density of the system through the logarithm of the diluteness parameter $n a^{2}$, where $n$ is the areal density of the disks and $a$ the hard-core diameter. Another important difference with respect to $3 \mathrm{D}$ systems is that while at zero temperature a fraction of the bosons undergo Bose-Einstein condensation, at finite temperature phase fluctuations destroy the longrange order (in agreement with the Mermin-Wagner theorem [3]) and the one-body density matrix decays algebraically to zero at large distances. The interacting 2D Bose gas is nevertheless a superfluid also at finite temperature and the nature of the transition is known 
to be of the Kosterlitz-Thouless type [4], the critical temperature being $T_{\mathrm{KT}} \approx \hbar^{2} n /\left(m \ln \ln \left(1 / n a^{2}\right)\right)$ in the ultra-dilute limit $\ln \ln \left(1 / n a^{2}\right) \gg 1$ [5]. In a recent experiment a 2D Bose gas has been realized using a film of gaseous Hydrogen on a Helium substrate [6].

A dilute 2D Bose gas inside a harmonic trap has also been considered by several authors. For the ideal gas the presence of the confinement introduces essential changes in the thermodynamic properties relative to the homogeneous case, and Bose-Einstein condensation starts at a finite temperature $k_{\mathrm{B}} T_{c} \approx \hbar \omega_{\perp} N^{1 / 2}$ where $\omega_{\perp}$ is the frequency of the $2 \mathrm{D}$ harmonic confinement and $N$ the number of particles [7]. The interacting system is expected to be Bose-condensed at low temperature $T<T_{\phi}$, but phase fluctuations should become important at a temperature $T_{\phi}$ where the phase correlation length becomes smaller than the radius of the cloud [8]. The precise behavior of the system at these temperatures and the nature of the phase transition have not yet been fully clarified (see, e.g., Fernandez and Mullin [9] and references therein).

In current experiments on Bose-Einstein condensates of alkali atoms in magnetic or optical traps the anisotropy of the confinement can be varied to obtain flatter and flatter (quasi-2D) condensates [10], with the ultimate possibility of observing the special features of low dimensionality. It is thus important to ascertain where in parameters space and which physical aspects of 2D systems will become dominant as the anisotropy is increased. The key question in this respect concerns which is the appropriate model for the boson-boson coupling strength in the regime of crossover between $3 \mathrm{D}$ and $2 \mathrm{D}$. A related issue is how to treat the coupling in a self-consistent calculation of the equilibrium properties of a Bose-Einstein condensed cloud.

In this Letter we evaluate this problem at zero temperature. We calculate the equilibrium density profiles of the condensate under different choices for its physical parameters, ranging from a 3D anisotropic system to a strictly 2D one. With increasing anisotropy the system first becomes 2D with regard to the confinement-only the lowest axial state is occupiedand then also in its collisional properties. We find that these different regimes can be identified by measuring the size of the cloud in the radial plane, and we characterize the cross-over in terms of the relevant physical parameters.
The Letter is organized as follows. In Section 2 we use existing theoretical results to introduce the coupling strengths describing the condensate in the transition from the $3 \mathrm{D}$ to the $2 \mathrm{D}$ regime and the corresponding non-linear Schrödinger equation (NLSE) in a local-density viewpoint. The results for the equilibrium density profile are given in Section 3, and Section 4 presents our conclusions and final remarks.

\section{Ground state and scattering properties of a quasi-2D condensate: a review}

We consider a dilute Bose-condensed gas at zero temperature under anisotropic (pancake-shaped) harmonic confinement characterized by the frequencies $\omega_{\perp}$ and $\omega_{z}=\lambda \omega_{\perp}$ with $\lambda \gg 1$. Within a densityfunctional approach, the ground-state properties of the gas are described in terms of the condensate wave function $\psi(r)$ in the $\{x, y\}$ plane, which is to be determined as the minimum of the local-density energy functional

$$
\begin{aligned}
E[\psi]= & \int d^{2} r\left[\frac{\hbar^{2}}{2 m}|\nabla \psi|^{2}+\left(V_{\mathrm{ext}}-\mu\right)|\psi|^{2}\right. \\
& \left.+\epsilon(n)|\psi|^{2}\right] .
\end{aligned}
$$

Here $n=|\psi|^{2}$ is the particle density, $\mu$ is the chemical potential, and $\epsilon(n)=g n / 2$ is the ground-state energy per particle of a homogeneous Bose gas with shortrange interactions in the mean-field approximation. The coupling $g$ can depend on the condensate density: $g \equiv g(\psi)$. The Euler equation $\delta E / \delta \psi^{*}=0$ leads to the NLSE for the condensate wave function ${ }^{1}$

$-\frac{\hbar^{2}}{2 m} \nabla^{2} \psi+V_{\text {ext }} \psi+g(\psi)|\psi|^{2} \psi=\mu \psi$.

The familiar Gross-Pitaevskii equation is recovered by taking a constant coupling $g$. Extensions of this equation beyond the mean field and local-density approximations have been proposed for systems at higher density [11-13].

We now need to specify the choice of $g$ for the system of present interest. Quite generally the cou-

\footnotetext{
${ }^{1}$ In the dilute-gas limit we also neglect the higher-order terms involving $\delta g / \delta \psi^{*}$.
} 
pling $g$ is obtained microscopically from the effective interaction potential $\Gamma\left(k, k^{\prime}, P\right)$ in the limit of low energy and momenta. The effective interaction can in turn be related to the two-body scattering function $f\left(\vec{k}, \vec{k}^{\prime}\right)=\int \psi_{\vec{k}}(r) v(r) e^{-i \vec{k}^{\prime} \cdot \vec{r}} d^{d} r$ in dimension $d=3$ or 2 , with $\psi_{\vec{k}}(r)$ being the outgoing wave function for the relative motion of the two particles and $v(r)$ being the interparticle potential [14]. For a 3D system $f\left(\vec{k}, \vec{k}^{\prime}\right)$ is the scattering amplitude and $g$ is a constant determined by the $s$-wave scattering length $a$ :

$g_{3 \mathrm{D}}=\frac{4 \pi \hbar^{2}}{m} a$.

On increasing the anisotropy of a 3D condensate, its physical properties will change first due to the modified shape of the confinement [15], but then also due to the modified scattering properties. We consider a condensate in a pancake-shaped trap which is flat enough so that the dimension of the cloud in the axial direction is of the order of the harmonic oscillator length $a_{z}=\sqrt{\hbar / m \omega_{z}}$. If the condition $a \ll a_{z}$ holds, the system experiences collisions in three dimensions and the coupling constant to be used in the 2D NLSE is

$g_{\mathrm{Q} 3 \mathrm{D}}=g_{3 \mathrm{D}}\left|\phi_{0}(0)\right|^{2}$

where $\phi_{0}(0)=\left(2 \pi a_{z}^{2}\right)^{-1 / 4}$ is the axial ground-state wave function evaluated at $z=0$. This coupling constant has been used by a number of authors (see, e.g., [16] and references therein). When the anisotropy further increases and $a$ approaches $a_{z}$, the collisions start to be influenced by the presence of the trap in the tight $z$ direction and the coupling $g$ in such a quasi-2D condensate has the form

$$
\begin{aligned}
g_{\mathrm{Q} 2 \mathrm{D}}= & g_{3 \mathrm{D}}\left|\phi_{0}(0)\right|^{2} \\
& \times \frac{1}{1+\frac{a}{a_{z} \sqrt{2 \pi}}\left|\ln \left(2(2 \pi)^{3 / 2} n a a_{z}\right)\right|} .
\end{aligned}
$$

This expression was originally derived by Petrov et al. $[8,17]$ by studying the scattering function of a system which is harmonically confined in the $z$ direction and homogeneous in the $\{x, y\}$ plane (see also footnote 18 in [8]). The coupling $g$ in this case depends on density, as is typical of 2D collisions.

Indeed, when the collisions are in the fully $2 \mathrm{D}$ regime $\left(a \gg a_{z}\right)$, the system is described by the coupling

$g_{2 \mathrm{D}}=\frac{4 \pi \hbar^{2}}{m} \frac{1}{\left|\ln \left(n a^{2}\right)\right|}$,

as was first derived by Schick for the homogeneous 2D system [1]. The use of the coupling $g_{2 \mathrm{D}}$ for a system under external confinement, involving a dependence on the local density, has been proposed by Shevchenko [18] and more recently by Kolomeisky et al. [19]. It was rigorously justified by Lieb and coworkers [16].

In the following section we will present results for the condensate wave function $\psi(r)$ as obtained from the NLSE (2) using the three couplings $g_{\mathrm{Q} 3 \mathrm{D}}, g_{\mathrm{Q} 2 \mathrm{D}}$ and $g_{2 \mathrm{D}}$.

\section{Equilibrium density profiles}

In current experiments on Bose-Einstein condensates of alkali atoms, one of the observables which are most directly accessible is the density profile. In particular, it is possible to take in situ images of the cloud, which can be directly compared with the results of theoretical models. We give in this section the predictions for the widths and the shapes of the equilibrium density in the $\{x, y\}$ plane for a condensate in a pancakeshaped trap under different collisional regimes as the anisotropy is increased.

Before proceeding to the numerical solution of the NLSE (2), it is useful to give a simple analytical estimate of the expected width of the cloud. We use for this purpose the Thomas-Fermi approximation, i.e., we neglect the kinetic-energy term in Eq. (2) to obtain the density profile

$$
\left|\psi_{\mathrm{TF}}(r)\right|^{2}=\frac{1}{\tilde{g}(\mu)}\left(\mu-V_{\mathrm{ext}}(r)\right) \theta\left(\mu-V_{\mathrm{ext}}(r)\right) .
$$

Here we have also neglected the spatial dependence of the coupling constant $\tilde{g}$ and used the result of the homogeneous system to relate the density to the chemical potential. ${ }^{2}$ Eq. (7) is valid when the number

\footnotetext{
2 The expressions for the coupling $\tilde{g}(\mu)$ that we actually use in this calculation are $\tilde{g}_{\mathrm{Q} 3 \mathrm{D}}(\mu)=2 \sqrt{2 \pi} \hbar^{2} a /\left(m a_{z}\right), \tilde{g}_{\mathrm{Q} 2 \mathrm{D}}(\mu)=$ $\left(4 \pi \hbar^{2} / m\right)\left(\sqrt{2 \pi} a / a_{z}+\ln \left(\lambda \hbar \omega_{\perp} / 2 \pi \mu\right)\right)^{-1}$ and $\tilde{g}_{2 \mathrm{D}}(\mu)=\left(4 \pi \hbar^{2} /\right.$ $m)\left|\ln \left(m \mu a^{2} / 4 \pi \hbar^{2}\right)\right|^{-1}$.
} 


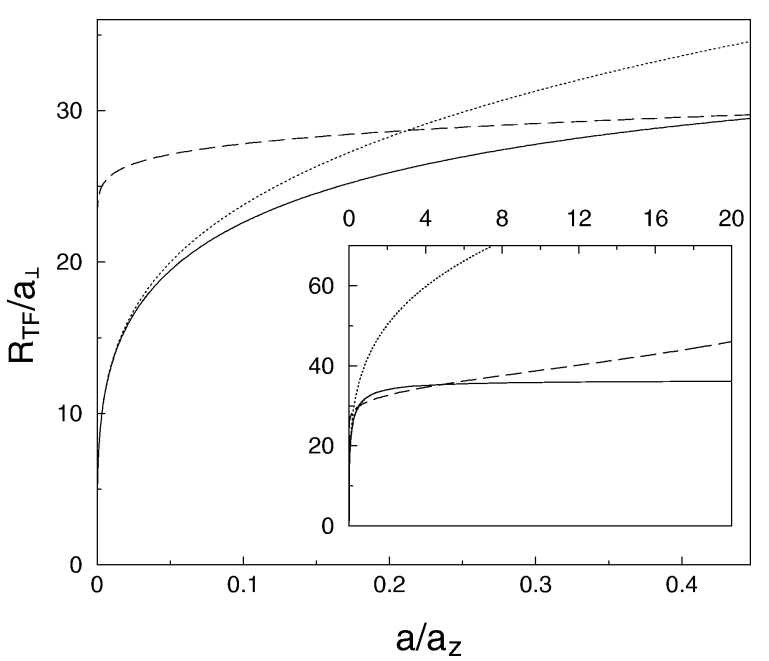

Fig. 1. Transverse width of the condensate (in units of $\left.a_{\perp}=\sqrt{\hbar / m \omega_{\perp}}\right)$ as a function of $a / a_{z}$ as evaluated in the Thomas-Fermi approximation for the three models Q3D (dotted line), Q2D (solid line) and 2D (dashed line). In the inset the same curves are plotted over a wide range of the ratio $a / a_{z}$. The system parameters are $N=5 \times 10^{5}$ atoms and $\lambda=2 \times 10^{5}$ for the trap anisotropy.

of atoms in the condensate is large and in this regime we have found that its predictions agree well with the full numerical solution of Eq. (2).

The chemical potential in Eq. (7) is fixed by imposing the normalization condition $N=\int\left|\psi_{\mathrm{TF}}(r)\right|^{2} d^{2} r$. By inversion of this equation we find an expression for the Thomas-Fermi width of the cloud, $R_{\mathrm{TF}}=$ $\sqrt{2 \mu / m \omega_{\perp}^{2}}$, in terms of the physical parameters of the system. We show in Fig. 1 the width of the cloud as a function of the ratio $a / a_{z}$ for a given choice of the number of atoms and of the anisotropy $\left(N=5 \times 10^{5}\right.$, $\left.\lambda=2 \times 10^{5}\right)$. We expect the quasi-3D model (4) for the coupling to be accurate only for small values for $a / a_{z}$. It evidently overestimates the size of the cloud as $a / a_{z}$ increases. The quasi-2D behavior should be correct for $a / a_{z}>0.1$ and is ultimately superseded by the purely $2 \mathrm{D}$ behavior as $a / a_{z}$ becomes appreciably larger than unity.

We now turn to the numerical solution of Eq. (2) with the alternative expressions (4)-(6) for the coupling. We have used the steepest descent method $[11,20]$, which is known to produce accurate results. A further check of our numerical calculations is provided by the virial relation.

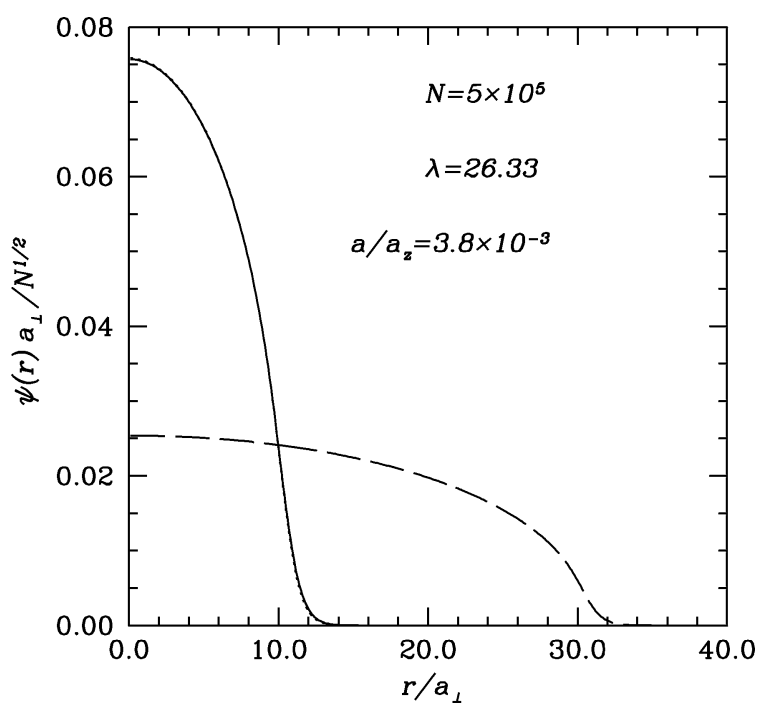

Fig. 2. Condensate wave function $\psi(r)$ (in units of $\sqrt{N} / a_{\perp}$ ) as a function of $r / a_{\perp}$, for $a / a_{z}=3.8 \times 10^{-3}$ from the full numerical solution of Eq. (2) for the three models Q3D (dotted line), Q2D (solid line) and 2D (dashed line). The values of the particle number and of the anisotropy parameter are indicated in the figure.

We give illustrations of the ground-state wave function $\psi(r)$ predicted by the three models in some relevant cases. First of all we have considered the values of particle number, anisotropy parameter, and scattering length as appropriate for ${ }^{23} \mathrm{Na}$ atoms in the experiment of Görlitz et al. [10] $\left(N=5 \times 10^{5}, \lambda=\right.$ 26.33, $a=2.8 \mathrm{~nm}$ ). In this case the system is approaching two-dimensionality for what concerns the confinement $\left(\mu \simeq 2.08 \hbar \omega_{z}\right)$, but still is $3 \mathrm{D}$ for collisions $\left(a / a_{z} \simeq 3.8 \times 10^{-3}\right)$. As is shown in Fig. 2, the quasi-3D and quasi-2D models give almost indistinguishable predictions (solid and dotted lines). The fully $2 \mathrm{D}$ model would give a quantitatively very different profile (dashed line in Fig. 2) with a much larger chemical potential $\left(\mu=17.8 \hbar \omega_{z}\right)$, but is evidently inapplicable in this regime of parameters.

For a second case we increase the anisotropy parameter to $\lambda=2 \times 10^{5}$ and make the choice $a / a_{z}=0.33$ for the scattering length. This corresponds to the point of cross-over in the scattering properties from 3D to 2D in a condensate where motion in the third direction is completely frozen by the confinement $(\mu \simeq$ $0.002 \hbar \omega_{z}$ ). In this case (Fig. 3) the three models predict comparable shapes of the cloud, which is an in- 


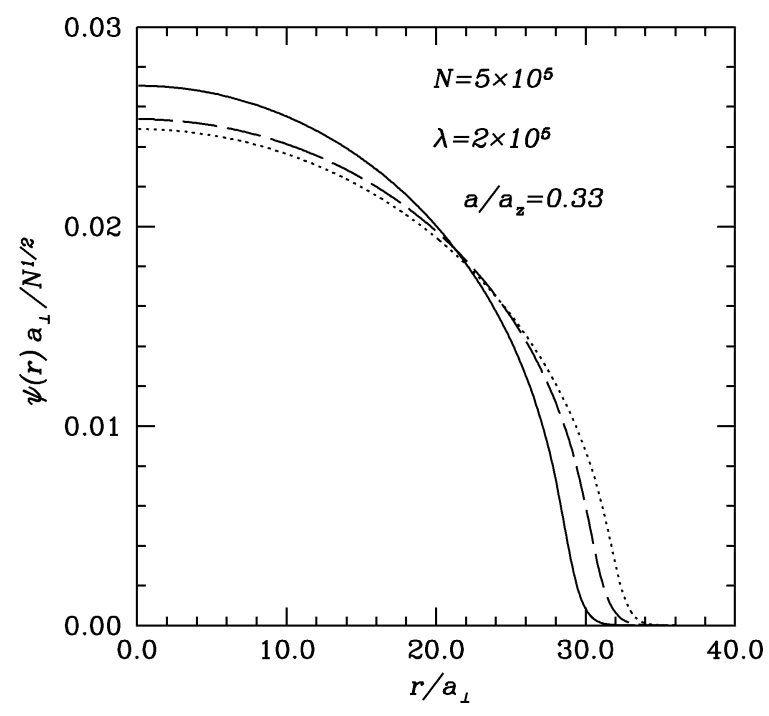

Fig. 3. The same as in Fig. 2, for $a / a_{z}=0.33$ and $\lambda=2 \times 10^{5}$.

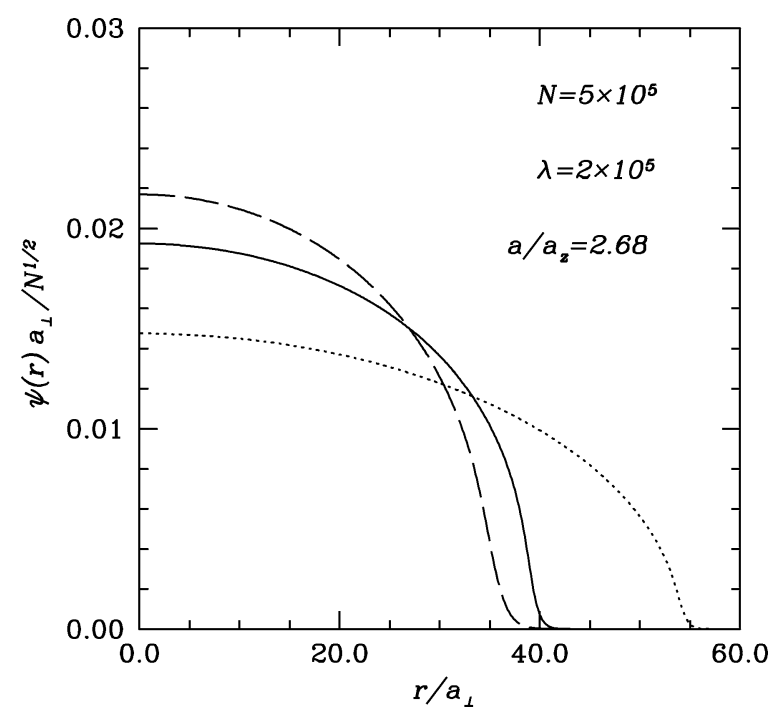

Fig. 4. The same as in Fig. 2, for $a / a_{z}=2.68$ and $\lambda=2 \times 10^{5}$.

dication that indeed the condensate is entering the regime of 2D collisions.

Finally, we have further increased the value of the scattering length to $a / a_{z} \simeq 2.68$, keeping the same values for $\lambda$ and $N$. For this choice of parameters the collisions are truly $2 \mathrm{D}$ and the fully $2 \mathrm{D}$ model should give the most accurate prediction for the shape of the cloud, while the other models overestimate its width
(Fig. 4). The calculated chemical potential of the $g_{2 \mathrm{D}}$ model is $\mu \simeq 0.0031 \hbar \omega_{z}$.

\section{Conclusions}

In summary, we have considered Bose-Einstein condensates confined inside pancake-shaped traps at zero temperature within a mean-field description. Depending on the anisotropy of the trap, three different physical regimes are identified for the scattering events:

(i) a quasi-3D regime where the axial dimension of the condensate is much larger than the scattering length and the collisions are as in a 3D condensate;

(ii) a quasi-2D regime where the tight harmonic confinement in the $z$ direction begins to influence the scattering events;

(iii) a fully $2 \mathrm{D}$ regime where collisions are restricted to the $\{x, y\}$ plane.

The atom-atom coupling is different in the three cases and a logarithmic dependence on the density arises as the 2D effects start to affect the scattering events. We have adopted a local-density approximation to introduce the appropriate atom-atom coupling into the energy functional of the condensate and to obtain a non-linear Schrödinger equation for its in-plane wave function.

Our main result of direct relevance to experiment is that the different collisional regimes are reflected in the width of the cloud. Its behaviour can be predicted with reasonable accuracy by the Thomas-Fermi model if the number of atoms in the condensate is not too low. We have also given some numerical illustrations based on solutions of the NLSE for various choices of parameters corresponding to the three collisional regimes. Our results for the fully 2D case are in accord with those reported recently by Lee et al. [21] by a numerical solution of the NLSE with the choice $\tilde{g}_{2 \mathrm{D}}(\mu)$ for the coupling strength.

In conclusion, the truly interesting properties that are expected for flat condensates should be observable when not only the condition $\mu<\hbar \omega_{z}$ is satisfied (the condensate is $2 \mathrm{D}$ for what concerns the confinement), but the condition $a / a_{z}>1$ also holds (the con- 
densate is $2 \mathrm{D}$ for what concerns the collisions). The strictly 2D limit can be reached in a pancake-shaped Bose-Einstein condensate by making the perpendicular confinement very tight and/or by increasing the scattering length, e.g., via Feshbach resonances. In order to understand the significance of interactions and high-density effects, high-precision Monte Carlo simulations would be useful as a test of the range of validity of the mean-field and local-density approximations. Our analysis should be the starting point for finite-temperature calculations, in which to study the role of the interactions with the non-condensed thermal particles and the transitions in phase correlations that are expected to occur with increasing temperature.

\section{Acknowledgements}

This work was partially supported by INFM under the project PRA2001-Photonmatter and under the PAIS2000 "Theory of two-dimensional interacting Bose gas". B.T. acknowledges support from the Scientific and Technical Research Council of Turkey (TUBITAK), NATO, the Turkish Department of Defense, and the Turkish Academy of Sciences (TUBA), and thanks Scuola Normale Superiore for hospitality during part of this work. A.M. acknowledges a travel grant from INFM under the initiative "Calcolo Parallelo".

\section{References}

[1] M. Schick, Phys. Rev. A 3 (1971) 1067.
[2] V.N. Popov, Functional Integrals in Quantum Field Theory and Statistical Physics, Reidel, Dordrecht, 1983, Chapter 6.

[3] N.D. Mermin, H. Wagner, Phys. Rev. Lett. 22 (1966) 1133.

[4] J.M. Kosterlitz, D.J. Thouless, J. Phys. C 6 (1973) 1181.

[5] D.S. Fisher, P.C. Hohenberg, Phys. Rev. B 37 (1988) 4936.

[6] A.I. Safonov, S.A. Vasilyev, I.S. Yasnikov, I.I. Lukashevich, S. Jaakkola, Phys. Rev. Lett. 81 (1998) 4545.

[7] R. Masut, W.J. Mullin, Amer. J. Phys. 47 (1979) 493; V. Bagnato, D. Kleppner, Phys. Rev. A 44 (1991) 7439; See also W.J. Mullin, J. Low Temp. Phys. 106 (1997) 615 and references therein.

[8] D.S. Petrov, M. Holzmann, G.V. Shlyapnikov, Phys. Rev. Lett. 84 (2000) 2551.

[9] J.P. Fernandez, W. Mullin, cond-mat/0203175.

[10] A. Görlitz, J.M. Vogels, A.E. Leanhardt, C. Raman, T.L. Gustavson, J.R. Abo-Shaeer, A.P. Chikkatur, S. Gupta, S. Inouye, T. Rosenband, W. Ketterle, Phys. Rev. Lett. 87 (2001) 130402.

[11] A. Fabrocini, A. Polls, Phys. Rev. A 60 (1999) 2319.

[12] G.S. Nunes, J. Phys. B 32 (1999) 4293.

[13] J.O. Andersen, H. Haugerud, Phys. Rev. A 65 (2002) 033615.

[14] A.A. Abrikosov, L.P. Gorkov, I.E. Dzyaloshinski, Methods of Quantum Field Theory in Statistical Physics, Prentice-Hall, Englewood Cliffs, 1963.

[15] L. Salasnich, A. Parola, L. Reatto, Phys. Rev. A 65 (2002) 043614. These authors have described the transition from a spherical to a pancake-shaped confinement at constant atomatom coupling.

[16] E.H. Lieb, R. Seiringer, J. Yngvason, Commun. Math. Phys. 224 (2001) 17.

[17] D.S. Petrov, G.V. Shlyapnikov, Phys. Rev. A 64 (2001) 012706.

[18] S.I. Shevchenko, Sov. Phys. JETP 73 (1991) 1009; Sov. J. Low Temp. Phys. 18 (1992) 223.

[19] E.B. Kolomeisky, T.J. Newman, J.P. Straley, X. Qi, Phys. Rev. Lett. 85 (2000) 1146.

[20] F. Dalfovo, S. Stringari, Phys. Rev. A 53 (1996) 2477.

[21] M.D. Lee, S.A. Morgan, M.J. Davis, K. Burnett, Phys. Rev. A 65 (2002) 043617. 